\title{
Hipoxemia y mecánica ventilatoria en pacientes con infección por coronavirus asociado a síndrome respiratorio agudo grave-2
}

Hypoxemia and respiratory mechanics in patients with severe acute respiratory syndrome-coronavirus 2 infection Hipoxemia e mecânica ventilatória em pacientes com infecção por coronavírus 2-síndrome respiratória aguda grave

Diego Andrés Ramírez Urízar, ${ }^{*}$ Alfredo Aisa Álvarez, ${ }^{*}$ Juvenal Franco Granillo, ${ }^{*}$ Janet Aguirre Sánchez, ${ }^{*}$ Uriel Elías Luján Sitt, ${ }^{*}$ María Vigil Escalera*

\section{RESUMEN}

Introducción: La pandemia de SARS-CoV-2 ha inspirado un nuevo interés en los fundamentos de la patología del síndrome de dificultad respiratoria aguda (SDRA) asociado con COVID-19. Este estudio busca las causas de hipoxemia y su mecánica ventilatoria en los pacientes en ventilación mecánica por COVID-19. Material y métodos: Estudio de cohorte prospectivo. Del 23 de marzo al 15 de mayo de 2020 se recolectó información basal (comorbilidades, estudios de laboratorio y escalas pronósticas) y parámetros ventilatorios y gasométricos de todos los pacientes mayores de 18 años que recibieron ventilación mecánica por COVID-19 y se dio seguimiento hasta el día 15 de ventilación mecánica (VM). Resultados: Un total de 63 pacientes, se excluyeron 13 por expedientes incompletos. Con distensibilidad pulmonar de $41.44 \pm 12.18 \mathrm{~mL} / \mathrm{cmH}_{2} \mathrm{O}$, driving pressure (DP) de $12.18 \pm 2.67 \mathrm{cmH}_{2} \mathrm{O}$, volumen tidal $(\mathrm{Vt})$ de $7.02 \pm 1.11 \mathrm{~mL} / \mathrm{kg}$ de peso predicho, cortocircuitos pulmonares de $23.38 \%$ (3.23-90.05), gradiente alveoloarterial $354.96 \pm 75.37 \mathrm{mmHg}$ y relación $\mathrm{PaO}_{2} / \mathrm{FiO}_{2}$ de 129 (48-309) $\mathrm{mmHg}$. El análisis lineal de medidas repetidas a la evaluación de los pacientes que presentaron extubación temprana versus VM prolongada, con diferencias estadísticamente significativas en Vt y DP $(p=0.04, p=0.0005$ respectivamente) si elevan más de $7.5 \mathrm{~mL} / \mathrm{kg}$ y $13 \mathrm{cmH}_{2} \mathrm{O}$ respectivamente.

Conclusiones: La hipoxemia en estos pacientes se debe a dos causas: aumento de cortocircuitos pulmonares y desequilibrio de ventilación/perfusión pulmonar. Se debe mantener una DP menor a $13 \mathrm{cmH}_{2} \mathrm{O}$ y un Vt menor a $7.5 \mathrm{~mL} /$ kg en todos los días de VM.

Palabras clave: Síndrome de distrés respiratorio del adulto, hipoxemia, ventilación mecánica, coronavirus.

\section{ABSTRACT}

Introduction: The SARS-CoV-2 pandemic has inspired new interest in the physiopathology of the acute respiratory distress syndrome (ARDS) due to COVID-19. This study describes the causes of hypoxemia and the respiratory mechanics in patients with COVID-19.

Material and methods: Prospective unicentric cohort study. Patient data recorded from march $23^{\text {rd }}$ till may $15^{\text {th }}$ of 2020 , we took basal data (comorbidities, laboratories and prognostic scales) and, ventilator and arterial blood gas data in patients aver 18 years of age which received mechanical ventilation (VM) due to COVID-19. We followed each patient for 15 days of mechanical ventilation. Results: A total of 50 patients, 13 were excluded due to incomplete data. The patients presented a mean of pulmonary compliance of $41.44 \pm 12.18 \mathrm{~mL}$ $\mathrm{CmH}_{2} \mathrm{O}$, driving pressure (DP) $12.18 \pm 2.67 \mathrm{cmH}_{2} \mathrm{O}$, tidal volume (Vt) $7.02 \pm$ $1.11 \mathrm{~mL} / \mathrm{kg}$ of predicted body weight, alveoloarterial gradient and $\mathrm{PaO} / \mathrm{FiO}_{2}$ relation 129 (48-309) $\mathrm{mmHg}$. We ran a lineal repeated measure analysis to evaluate differences in mechanical ventilation on patients who where extubated early (Et) versus prolonged VM. With statistical significant differences with $V t$ and $D P(p=0.04, p=0.0005$ respectively) when they elevate more than $7.5 \mathrm{~mL}$ kg y $13 \mathrm{cmH}_{2} \mathrm{O}$ respectively.

Conclusions: hypoxemia in mechanically ventilated patients due to augmented intrapulmonary shunts and ventilation/perfusion mismatch. In these patients throughout every day of VM, the Vt less than $7.5 \mathrm{~mL} / \mathrm{kg}$ and DP less than $13 \mathrm{cmH}_{2} \mathrm{O}$. Keywords: Acute respiratory distress syndrome, hypoxemia, mechanical ventilation, coronavirus.

\section{* Centro Médico ABC. México.}

Recibido: 16/10/2020. Aceptado: 09/11/2020.

Citar como: Ramírez UDA, Aisa ÁA, Franco GJ, Aguirre SJ, Luján SUE, Vigil EM. Hipoxemia y mecánica ventilatoria en pacientes con infección por coronavirus asociado a síndrome respiratorio agudo grave-2. Med Crit. 2021;35(1):10-17. https://dx.doi. org/10.35366/99148

www.medigraphic.com/medicinacritica
RESUMO

Introdução: A pandemia SARS-CoV-2 inspirou novo interesse nos fundamentos da patologia da síndrome da dificuldade respiratória do adulto (SDRA) associada ao COVID-19. Este estudo busca as causas da hipoxemia e sua mecânica ventilatória em pacientes em ventilação mecânica por COVID-19. Material e métodos: Estudo de coorte prospectivo. De 23 de março a 15 de maio de 2020, informações basais (comorbidades, estudos laboratoriais e escalas de prognóstico) e parâmetros ventilatórios e gasométricos foram coletados de todos os pacientes com mais de 18 anos que receberam ventilação mecânica para COVID-19 e acompanhados até o dia 15 de ventilação mecânica (VM).

Resultados: Um total de 63 pacientes, 13 pacientes foram excluídos devido a prontuários incompletos. Com complacência pulmonar de $41.44 \pm 12.18 \mathrm{~mL} /$ $\mathrm{CmH}_{2} \mathrm{O}$, driving pressure (DP) de $12.18 \pm 2.67 \mathrm{cmH}_{2} \mathrm{O}$, volume corrente $(\mathrm{Vt}) \mathrm{de}$ $7.02 \pm 1.11 \mathrm{~mL} / \mathrm{kg}$ de peso previsto, shunts pulmonares de $23.38 \%$ (3.23-90.05), gradiente alveolo arterial $354.96 \pm 75.37 \mathrm{mmHg}$ e relação $\mathrm{PaO}_{2} / \mathrm{FiO}_{2}$ de 129 (48309) $\mathrm{mmHg}$. A análise linear de medidas repetidas para avaliação de pacientes que apresentavam extubação precoce versus VM prolongada, com diferenças estatisticamente significativas no Vt e DP ( $p=0.04, p=0.0005$ respectivamente) quando eles elevam mais de $7.5 \mathrm{~mL} / \mathrm{kg}$ e $13 \mathrm{cmH}_{2} \mathrm{O}$ respectivamente.

Conclusões: A hipoxemia nesses pacientes deve-se a duas causas: aumento dos shunts pulmonares e desequilíbrio ventilação/perfusão pulmonar. Um DP menor que $13 \mathrm{cmH}_{2} \mathrm{O}$ e um Vt menor que $7.5 \mathrm{~mL} / \mathrm{kg}$ devem ser mantidos em todos os dias de VM.

Palavras-chave: Síndrome da dificuldade respiratória do adulto, hipoxemia, ventilação mecânica, coronavírus.

\section{INTRODUCCIÓN}

La pandemia de SARS-CoV-2 ha inspirado un nuevo interés en los fundamentos de la patología del síndrome de dificultad respiratoria aguda (SDRA) que se ha asociado con COVID-19. EI SDRA se ha reconocido como una enfermedad heterogénea, no sólo con múltiples causas, sino con un espectro amplio de gravedad, anormalidades en imagenología y disfunción en el intercambio gaseoso. La forma de SDRA asociado con COVID-19 no es diferente. ${ }^{1}$

En un estudio llevado a cabo con 38 pacientes que fallecieron por COVID-19 en dos hospitales del norte de Italia, se encontró que todos los casos presentaron fase exudativa y proliferativa de daño alveolar, el cual incluía: congestión capilar (100\%), necrosis de los neumocitos (100\%), membranas hialinas (33\%), edema intersticial e intraalveolar (37\%), hiperplasia de los neumocitos tipo 2 (100\%), metaplasia escamosa con atipia (21\%) y trombosis (33\%). Los pacientes con COVID-19 presentan lesión endotelial, asociada con la presencia intracelular del virus que causa ruptura de la membrana celular. Existe microtrombosis de los capilares alveolares y microangiopatía. El infiltrado inflamatorio (100\%) se compone principalmente de macrófagos en la mem- 
brana alveolar y linfocitos en el intersticio. La microscopia electrónica reveló que las partículas virales se encontraron alojadas principalmente en los neumocitos. ${ }^{2}$

Ackerman y colaboradores elucidaron algunos mecanismos biológicos que pueden resultar en SDRA por COVID-19. Los investigadores realizaron un estudio histológico detallado en pulmones de pacientes con COVID-19 y muestras históricas de pacientes con influenza H1N1 del año 2009. Ambos grupos presentaron daño alveolar difuso con signos de trombosis. Este hallazgo en el alveolo es patognomónico para SDRA, con micro y macrotrombosis presente. Sin embargo, Ackerman y su equipo encontraron niveles aumentados de angiogénesis en pacientes con SDRA por COVID-19. ${ }^{3}$

Los datos clínicos principales de los pacientes con daño pulmonar secundario a enfermedad por COVID-19 son los siguientes:

1. Hipoxemia grave.

2. Isocapnia.

3. Buena distensibilidad pulmonar.

4. Curso prolongado de la enfermedad.

5. Infección bacteriana secundaria poco frecuente $(6.9 \%) .{ }^{4}$

Varios estudios han reportado las manifestaciones clínicas de COVID-19, de los primeros reportes de casos en Wuhan, Chen y colaboradores reportaron que $82 \%$ de los pacientes presentó tos, $31 \%$ disnea y $5 \%$ dolor faríngeo. De éstos, $75 \%$ presentó neumonía bilateral y $1 \%$ presentó SDRA. ${ }^{5}$ A pesar de conocer el cuadro clínico, nuestro entendimiento del compromiso pulmonar no es claro. Li y su grupo de investigadores ${ }^{6}$ generaron la duda sobre las diferencias del SDRA por COVID-19 con las definiciones de los criterios de Berlín. ${ }^{7}$ Hacen hincapié en el tiempo de presentación clínica (media de 12 días), las distensibilidades pulmonares normales/elevadas y las implicaciones terapéuticas de la relación $\mathrm{PaO}_{2} / \mathrm{FiO}_{2}$ menor a 150. Gattinoni y colaboradores ${ }^{8}$ proponen dos fenotipos de neumonía por COVID-19: el tipo 1, distensibilidades pulmonares casi normales (mayores de $50 \mathrm{~mL} / \mathrm{cmH}_{2} \mathrm{O}$ ), neumonía viral e hipoxemia grave por desequilibrio en la ventilación/ perfusión; y el tipo 2, distensibilidades pulmonares bajas (menor a $40 \mathrm{~mL} / \mathrm{cmH}_{2} \mathrm{O}$ ) con hipoxemia severa por cortocircuitos pulmonares. Hay que tomar en cuenta la evolución cronológica de la enfermedad, ya que el mismo paciente puede presentar los mismos fenotipos.

En un gran estudio de cohorte español $(n=742)$, en el cual se evaluaron las características clínicas de los pacientes graves con COVID-19, se concluyó que presentan características fisiológicas (distensibilidad de sistema respiratorio (Crs), presión plateau (Pplat) y driving pressure) similares a las causadas por SDRA, secundario a otras causas, y que la probabilidad de morir incrementa con el grado de SDRA que presenten los pacientes. Interesantemente, se encontró una significativa redistribución de la gravedad de los pacientes con SDRA a las 24 horas de diagnóstico. Se cree que esta disminución de la gravedad fue secundaria al uso de ventilación mecánica con presión positiva y al tratamiento adyuvante. ${ }^{9}$

El objetivo principal de este estudio es documentar el comportamiento de la ventilación mecánica y de los parámetros gasométricos durante la ventilación mecánica en pacientes con SDRA por SARS-CoV-2, y asociar factores de ventilación mecánica y gasométricos con eventos no deseados en pacientes con ventilación mecánica (ventilación mecánica prolongada, muerte, reintubación/falla a la extubación).

\section{MATERIAL Y MÉTODOS}

Diseño del estudio: este es un estudio prospectivo de cohorte de pacientes mayores de 18 años, quienes ingresaron a terapia intensiva, con requerimiento de ventilación mecánica en el periodo del 23 de marzo al 15 de mayo de 2020. El estudio se aprobó por el comité de ética del centro asistencial, Ciudad de México. Este estudio se reporta según las guías Strengthening the Reporting of Observational Studies in Epidemiology (STROBE, por sus siglas en inglés). ${ }^{10}$

Población del estudio y colección de datos: los datos de los casos fueron tomados de expedientes electrónicos y expedientes en físico por médicos entrenados en medicina crítica con un protocolo estandarizado de colección de datos. Cada investigador tiene acceso a los expedientes electrónicos (TIMSA) y se recolectaron los expedientes en físico para los datos faltantes. Se aseguró que los expedientes hubieran cumplido cuarentena y que tuvieran adecuada esterilización después de haber sido utilizados en el área de atención de pacientes infectados por SARS-CoV-2. Se registraron todos los pacientes con las siguientes características: mayores de 18 años, intubados y con ventilación mecánica, con infección por SARS-CoV-2 confirmada por PCR del tracto respiratorio y que presentaran SDRA por criterios de Berlín, que incluye nuevos o empeoramiento de síntomas respiratorios por infección por COVID-19, infiltrados pulmonares bilaterales por imagenología (radiografía o tomografía de tórax), ausencia de hipertensión del atrio izquierdo o sin hallazgos de falla cardiaca izquierda e hipoxemia. Se define hipoxemia como la relación entre la presión parcial de oxígeno y la fracción inspirada de oxígeno $\left(\mathrm{PaO}_{2} / \mathrm{FiO}_{2}\right) \leq 300 \mathrm{mmHg}$, con una presión positiva al final de espiración (PEEP, por sus siglas en inglés) $\geq 5 \mathrm{cmH}_{2} \mathrm{O}$ sin tomar en cuenta el $\mathrm{FiO}_{2}$. Los criterios de exclusión fueron casos con infección no confirmada por SARS-CoV-2 según las guías de la OMS, los pacientes sin datos basales, sin infor- 
mación de los parámetros ventilatorios o gasométricos y no intubados.

Los datos tomados en el estudio incluyen: datos demográficos (edad, sexo, peso, peso predicho ARDSNet, índice de masa corporal [IMC], comorbilidades), parámetros de laboratorio (leucocitos, linfocitos, dímero D, proteína $C$ reactiva, procalcitonina, ferritina, interleucina-6, plaquetas), parámetros gasométricos $(\mathrm{pH}$, presión parcial de oxígeno arterial $\left[\mathrm{PaO}_{2}\right]$, presión parcial de oxígeno venoso $\left[\mathrm{PvO}_{2}\right]$, presión parcial de dióxido de carbono arterial $\left[\mathrm{PaCO}_{2}\right]$, presión parcial de dióxido de carbono venoso $\left[\mathrm{PvCO}_{2}\right]$, saturación arterial de oxígeno $\left[\mathrm{SaO}_{2}\right]$ y saturación venosa central de oxígeno $\left[\mathrm{SvcO}_{2}\right]$, bicarbonato, exceso/déficit de base), parámetros ventilatorios (volumen tidal, fracción inspirada de oxígeno $\left[\mathrm{FiO}_{2}\right]$, frecuencia respiratoria [FR], presión positiva al final de la inspiración [PEEP, por sus siglas en inglés], presión meseta y distensibilidad pulmonar), días de ventilación mecánica, días en terapia intensiva, días de hospitalización, evaluación secuencial de falla orgánica (SOFA, por sus siglas en inglés), APACHE II, SAPS II y falla a la extubación/reintubación.

Los datos completos de los pacientes fueron tomados el día 1 de ventilación mecánica y los datos de laboratorio, gasométricos y parámetros ventilatorios se tomaron desde el día 1 de ventilación mecánica hasta el día de la primera extubación, muerte o hasta 30 días después del 15 de mayo. Todos los parámetros gasométricos y ventilatorios se tomaron en el periodo de las 5:00-6:00 horas de todos los días, con $\mathrm{FiO}_{2}$ al $100 \%$ como protocolo de atención de los pacientes del centro de estudio. Dos investigadores valoraron la base de datos para errores, se validaron resultados y se corrigieron errores.

Análisis estadístico: para el objetivo principal, se dividió la cohorte en dos grupos, extubación temprana (menor a 14 días) y ventilación mecánica prolongada (mayor a 14 días). ${ }^{11}$ Al dividir los dos grupos, describimos las características clínicas, datos de ventilación mecánica, parámetros ventilatorios y gasométricos. Las variables descriptivas se expresan como porcentajes, media y desviación estándar o mediana con rangos intercuartiles, según sea apropiado. Después, comparamos las variables entre los grupos, usando suma de rangos de Wilcoxon o t de Student para variables numéricas y la prueba exacta de Fisher para variables categóricas. Posteriormente, decidimos buscar la relación de la mecánica ventilatoria durante los días de ventilación mecánica con la ventilación mecánica prolongada. Al ser medidas que se toman de forma diaria, se realizó análisis lineal de medidas repetidas para driving pressure, volumen tidal y PEEP. No tomamos en cuenta la distensibilidad pulmonar ya que se traduce en driving pressure.

Como estudio observacional, no presentó beneficio o daño a los pacientes. Nuestra meta fue evaluar a to- dos los ingresados a nuestro centro, quienes recibieron ventilación mecánica por infección por SARS-CoV-2, sin ningún tamaño de muestra predefinido. Se definió como día 1 el primer día de ventilación mecánica invasiva. Los datos faltantes no fueron imputados. Todos los análisis fueron de dos grupos, y un valor de $p<0.05$ se consideró estadísticamente significativo. Todos los análisis se realizaron con STATA versión 16.

\section{RESULTADOS}

Características basales: en un periodo de 54 días, se ingresaron 63 pacientes a la Unidad de Cuidados Intensivos (UCI) del centro asistencial, y se les dio seguimiento durante un mínimo de 30 días a todos ellos. De los 63 casos, se excluyeron 13 , ocho no tenían parámetros ventilatorios completos y cinco no tenían parámetros gasométricos completos. Se analizaron 50 pacientes desde el día 1 de ventilación mecánica, hasta su extubación o cumplir criterios de ventilación mecánica prolongada (> 14 días). El seguimiento de los casos permaneció después de los 30 días, ya que tres pacientes $(6 \%)$ permanecían en terapia intensiva. Los datos demográficos, comorbilidades, escalas APACHE II, SAPS II y SOFA, estudios de laboratorio, días de ventilación mecánica, días en la UCI y días de hospitalización se pueden visualizar en la Tabla 1. En el periodo de recolección de datos se documentó $18 \%$ de pacientes que cumplieron criterios para ventilación mecánica prolongada. Se hace notar en las características basales que los casos presentaron distensibilidades pulmonares de $41.44 \pm 12.18$ $\mathrm{mL} / \mathrm{cmH}_{2} \mathrm{O}$, driving pressure de $12.18 \pm 2.67$ y volumen corriente de $7.02 \pm 1.11$; al comparar la revisión de Ferrando y colaboradores ${ }^{9}$ la driving pressure y el volumen corriente son muy similares, con los pacientes presentando distensibilidades pulmonares mayores. En promedio, los casos presentaron elevación de cortocircuitos pulmonares $23.38 \%$ (3.23-90.05), aumento del gradiente alveoloarterial $354.96 \pm 75.37 \mathrm{mmHg}$, con un promedio de relación $\mathrm{PaO}_{2} / \mathrm{FiO}_{2}$ de 129 (48-309) mmHg. Al valorar los dos grupos de la cohorte (extubados de forma temprana vs ventilación mecánica prolongada), en cuanto a las características demográficas, se notó diferencia estadísticamente significativa en edad (Tabla 2), no se encontraron diferencias en escalas pronósticas o parámetros de laboratorio. Se notan diferencias en días de ventilación mecánica, días en la UCI y días de hospitalización; todas las muertes (tres pacientes) se dieron en el grupo de ventilación mecánica prolongada.

Causas de hipoxemia y parámetros gasométricos: al inicio de la ventilación mecánica, se encontraron hipoxémicos, con una relación $\mathrm{PaO}_{2} / \mathrm{FiO}_{2}$ de 129 (48-309) mmHg y normocápnicos, con $\mathrm{PaCO}_{2}$ de 41 (22-71) mmHg (Tabla 1), con saturaciones venosas centrales elevadas $80.11 \pm 9.85 \%$ y diferencia venoarterial de $\mathrm{CO}_{2}$ normales 
Tabla 1: Características clínicas y demográficas de los pacientes.

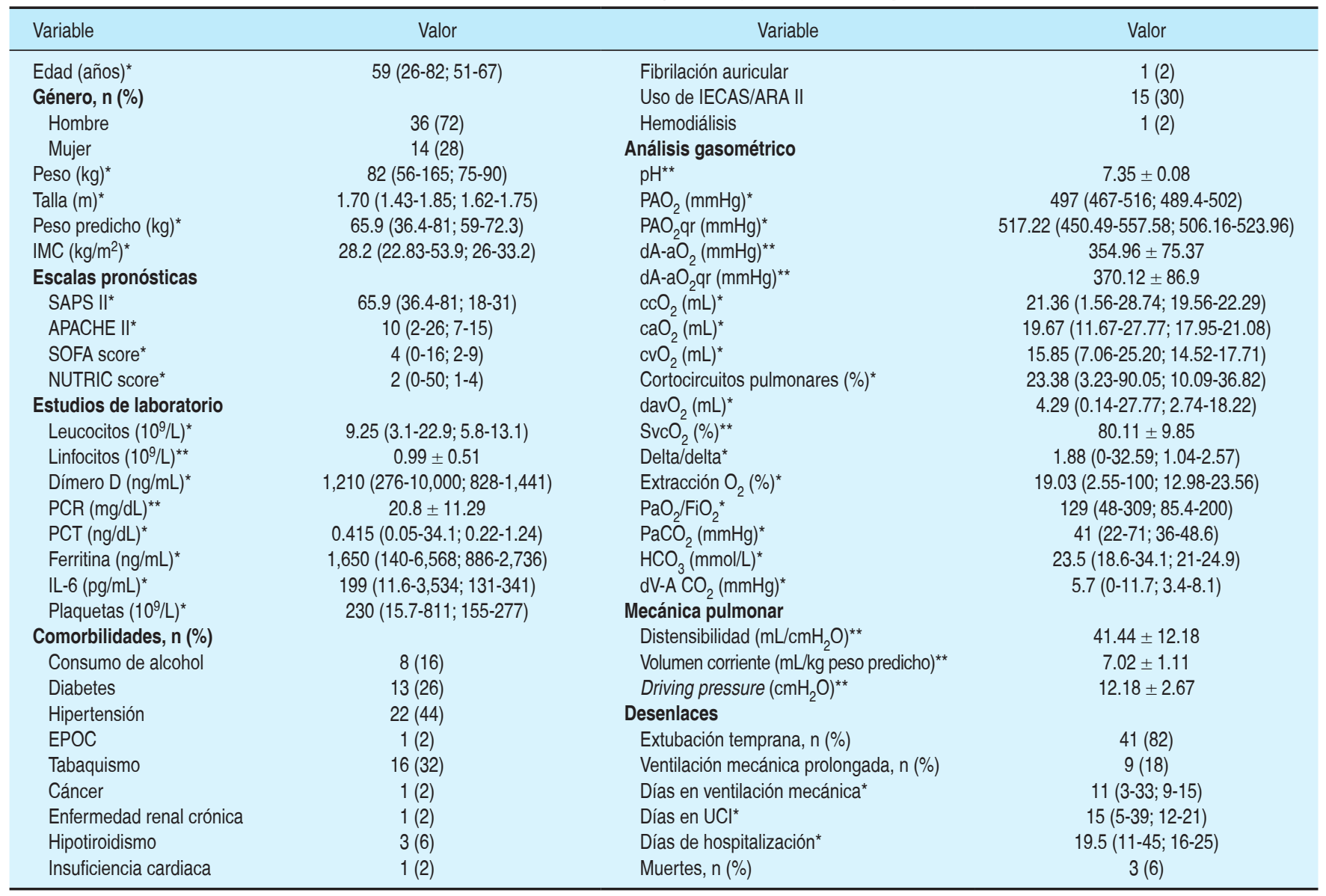

* Mediana (mín.-máx.; p25-p75). ** Media \pm DE.

SAPS II = Simplified Acute Physiology Score II; APACHE II = Acute Physiology Age Chronic Health Evaluation; SOFA = Sequential Organ Failure Assessment; NUTRIC score = Nutrition Risk in Critically III score; PCR = proteína C reactiva; $\mathrm{PCT}=$ procalcitonina; IL-6 = interleucina $6 ; \mathrm{EPOC}=$ enfermedad pulmonar obstructiva crónica; IECAS/ARA II $=$ inhibidores de la enzima convertidora de angiotensina/antagonistas de los receptores de la angiotensina II; $\mathrm{PAO}_{2}=$ presión alveolar de oxígeno; $\mathrm{PAO}_{2} \mathrm{qr}=$ presión alveolar de oxígeno corregida; $\mathrm{dA}-\mathrm{aO}_{2}=$ gradiente alveoloarterial de oxígeno; $\mathrm{dA}-\mathrm{O}_{2} \mathrm{qr}=$ gradiente alveoloarterial de oxígeno corregido; $\mathrm{ccO}_{2}=\mathrm{contenido}$ capilar de oxígeno; caO = contenido arterial de oxígeno; $\mathrm{cvO}_{2}=$ contenido venoso de oxígeno; davO $\mathrm{O}_{2}=$ diferencia arteriovenosa de oxígeno; $\mathrm{SvcO}_{2}=$ saturación venosa central de oxígeno; $\mathrm{PaO} \mathrm{O}_{2} \mathrm{~J}$ $\mathrm{FiO}_{2}=$ relación presión parcial de oxígeno arterial/fracción inspirada de oxígeno; $\mathrm{PaCO}_{2}=$ presión parcial de dióxido de carbono arterial; $\mathrm{HCO}_{3}=$ bicarbonato; dV-A CO $2=$ diferencia venoarterial de dióxido de carbono; $\mathrm{UCl}$ = Unidad de Cuidados Intensivos.

$5.7(0-11.7) \mathrm{mmHg}$. El pH al ingreso de los pacientes era normal, con una media de $7.35 \pm 0.08$. Se hace notar que al inicio de la ventilación mecánica, los pacientes presentaron elevación del gradiente alveoloarterial (354.96 $\pm 75.37 \mathrm{mmHg}$ ), así como el gradiente corregido por la relación delta/delta (diferencia arteriovenosa de contenido de oxígeno/diferencia venoarterial de $\left.\mathrm{CO}_{2}\right)(370.12 \pm 86.9$ $\mathrm{mmHg}$ ) y elevación de cortocircuitos pulmonares con una mediana de $23.38 \%$ (10.09-36.82). Al analizar a los sujetos por los dos diferentes grupos, se nota quienes presentaron diferencias en el $\mathrm{pH}$ al ingreso $(7.37 \pm 0.07 \mathrm{vs}$ $7.28 \pm 0.10$ ), además de diferencias no estadísticamente significativas, pero de importancia clínica, con el contenido arterial de oxígeno $\left(\mathrm{caO}_{2}\right)(19.74 \mathrm{~mL}$ vs $17.88 \mathrm{~mL})$ y de cortocircuitos pulmonares (20.9 vs $33.24 \%$ ).

Parámetros ventilatorios: aquéllos de importancia para pacientes con SIRA ${ }^{12-14}$ se analizaron por me- dio de análisis lineal del medidas repetidas (Figura 1), tomando en cuenta el volumen tidal $(\mathrm{Vt})$, la presión positiva al final de la inspiración (PEEP por sus siglas en inglés) y la driving pressure (DP). Se evaluó la evolución cronológica desde el día 1 hasta el día 15 de la ventilación mecánica. Se hace notar que hay cambios estadísticamente significativos en la evolución de la cantidad de Vt brindado al paciente y de la DP. Con diferencias estadísticamente no significativas en la cantidad de PEEP brindado a los pacientes. El comportamiento de la DP de forma cronológica (Figura 2) denota que en los casos del grupo de extubación temprana (Et), la DP en los primeros días nunca subió de $13 \mathrm{cmH}_{2} \mathrm{O}$, mientras que en los pacientes del grupo de VM prolongada, a partir del día 15, el promedio de la DP fue mayor a $13 \mathrm{cmH}_{2} \mathrm{O}$, con hallazgos estadísticamente significativos. 
Tabla 2: Características clínicas y demográficas por grupos de extubación temprana y ventilación mecánica prolongada.

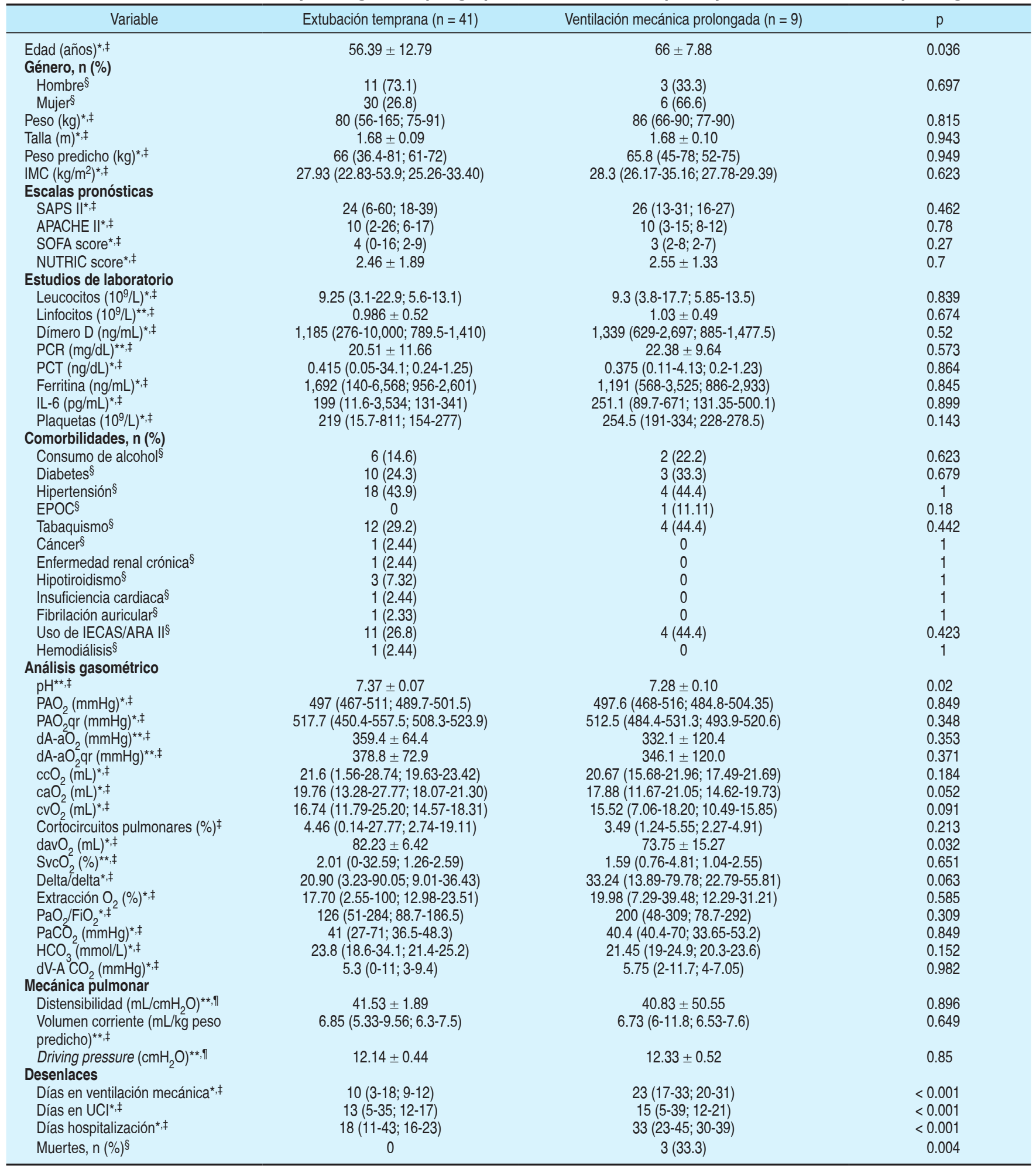

\footnotetext{
${ }^{*}$ Mediana (mín.-máx.; p25-p75). ** Media \pm DE. $¥$ Suma de rangos de Wilcoxon. § Prueba exacta de Fisher. 9 t Student.
}

SAPS II = Simplified Acute Physiology Score II; APACHE II = Acute Physiology Age Chronic Health Evaluation; SOFA = Sequential Organ Failure Assessment; NUTRIC score = Nutrition Risk in Critically III score; PCR = proteína C reactiva; PCT = procalcitonina; IL-6 = interleucina 6; EPOC = enfermedad pulmonar obstructiva crónica; IECAS/ARA II $=$ inhibidores de la enzima convertidora de angiotensina/antagonistas de los receptores de la angiotensina II; $\mathrm{PAO}_{2}=$ presión alveolar de oxígeno; $\mathrm{PAO}_{2} \mathrm{qr}=$ presión alveolar de oxígeno corregida; $\mathrm{dA}-\mathrm{aO}_{2}=$ gradiente alveoloarterial de oxígeno; $\mathrm{dA}-\mathrm{O}_{2} \mathrm{qr}=$ gradiente alveoloarterial de oxígeno corregido; $\mathrm{ccO}_{2}=\mathrm{contenido}_{2}$ capilar de oxígeno; caO $=$ contenido arterial de oxígeno; $\mathrm{CvO}_{2}=$ contenido venoso de oxígeno; davO $\mathrm{O}_{2}=$ diferencia arteriovenosa de oxígeno; $\mathrm{SvcO}_{2}=$ saturación venosa central de oxígeno; $\mathrm{PaO} \mathrm{O}_{2} \mathrm{I}$ $\mathrm{FiO}_{2}=$ relación presión parcial de oxígeno arterial/fracción inspirada de oxígeno; $\mathrm{PaCO}_{2}=$ presión parcial de dióxido de carbono arterial; $\mathrm{HCO}_{3}=$ bicarbonato; dV- $\mathrm{A} \mathrm{CO}_{2}=$ diferencia venoarterial de dióxido de carbono; $\mathrm{UCl}=$ Unidad de Cuidados Intensivos. 


\section{DISCUSIÓN}

En este estudio unicéntrico, observacional de 50 pacientes bajo ventilación mecánica por SDRA por infección por SARS-CoV-2, se encuentran pacientes predominantemente mayores de 50 años, masculinos, con sobrepeso y condiciones comórbidas. Presentaron medias de ventilación mecánica de 11 días, 15 días en la UCl y 19.5 días de hospitalización. En promedio con criterios para SDRA moderado por criterios de Berlín, ${ }^{7}$ con características muy similares de los parámetros ventilatorios y mecánica pulmonar basal documentadas por Ferrando y colaboradores ${ }^{9}$ en pacientes con SDRA por COVID-19. Se documentó una mortalidad de $6 \%$ en los 84 días de seguimiento de los pacientes, tomando en cuenta que permanecían tres con ventilación mecánica prolongada al terminar el seguimiento. Como se reportó antes para pacientes con COVID-19, las comorbilidades más frecuentes fueron hipertensión, diabetes y sobrepeso/obesidad. $9,15,16$

En promedio, los casos recibieron ventilación mecánica con protección alveolar, con volúmenes corrientes de $7.02 \pm 1.11 \mathrm{~mL} / \mathrm{kg}$ de peso predicho, presiones meseta menores a $30 \mathrm{cmH}_{2} \mathrm{O}$ y DP de $12.18 \pm 2.67 \mathrm{cmH}_{2} \mathrm{O}$. La alta prevalencia de mantener ventilación mecánica protectora se puede explicar con el hecho de que desde el ingreso a la UCI tenían el diagnóstico de SIRA, el estudio LUNG SAFE ${ }^{17}$ documenta ventilación no protectora en quienes no se había sospechado SDRA previamente. Estudios previos han elevado la sospecha de las causas de hipoxemia en pacientes con SDRA por infección por SARS-CoV-2, ${ }^{6,8}$ nosotros documentamos que los casos al inicio de la ventilación mecánica ya presentan elevación de cortocircuitos pulmonares (media de $23.38 \%$ ), así como trastornos de ventilación perfusión (dA-aO2qr de $370.12 \pm 86.9$ ). Decidimos corregir el gradiente alveoloarterial, ya que los pacientes no se encuentran en un estado estable ${ }^{18}$ su cociente respiratorio probablemente no es de $0.8 .{ }^{19} \mathrm{Al}$ evaluar a los pacientes en los grupos de Et y VM prolongada, se encuentran diferencias como factores determinantes para presentar posteriormente VM prolongada, como la edad, que siempre ha influenciado en el pronóstico del paciente. ${ }^{20}$ En el análisis gasométrico comparativo se nota la diferencia de $\mathrm{pH}$ entre los grupos $(7.37 \pm 0.07$ vs $7.28 \pm 0.10$ ) estadísticamente significativa, sin diferencias en niveles de bicarbonato $\circ \mathrm{PaCO}_{2}$, indicando que la acidemia independiente del origen se asocia con VM prolongada a la presentación clínica. Existen diferencias entre los grupos de Et y VM prolongada en la cantidad de cortocircuitos pulmonares (20.9 vs $33.24 \%$ respectivamente) con una $p=0.052$, aunque no es estadísticamente significativo, es clínicamente significativo, se tendrían que estudiar en muestras más grandes. El mayor grado de cortocircuitos pulmonares en pacien-
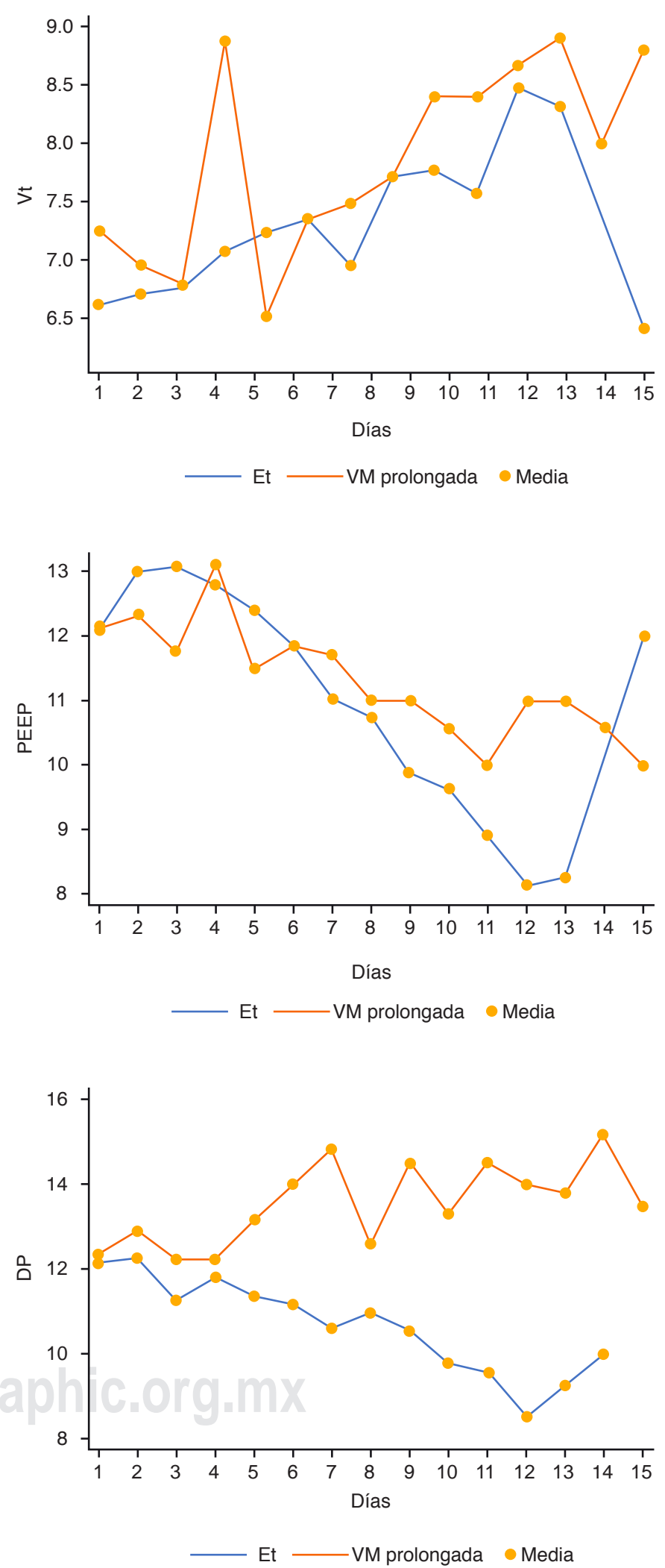

Figura 1: Comportamiento de parámetros ventilatorios en los pacientes con extubación temprana versus ventilación mecánica prolongada.

$\mathrm{Vt}=$ volumen tidal; PEEP = presión positiva al final de la inspiración; VM prolongada = ventilación mecánica prolongada; Et = extubación temprana; $\mathrm{DP}=$ driving pressure. 
tes con SDRA por COVID-19 se asocia con la evolución tardía de la enfermedad. ${ }^{6,8,21}$ Puede que exista un rol de la fórmula de cortocircuitos pulmonares en pacientes con SDRA por COVID-19 descrita por Riley y su grupo en el año $1951 .^{22}$

Existe una relación estadísticamente significativa en el comportamiento del Vt y la DP de forma cronológica y VM prolongada, no se encuentra la misma relación estadísticamente significativa para el nivel de PEEP. Se encuentra un cambio de Vt y DP posterior al cuarto día de ventilación mecánica en el análisis multivariable lineal de medidas repetidas, lo mismo se logra visualizar en los niveles de PEEP. Ya se conoce que el Vt y la DP juegan un papel importante en el pronóstico de los pacientes bajo ventilación mecánica por SDRA. ${ }^{12,14}$ Hacemos hincapié en que estos resultados son independientes a características heterogéneas de los grupos Et y VM prolongada. La evolución cronológica del Vt y de la DP es sumamente importante en pacientes con SDRA por COVID-19, independiente de la causa de hipoxemia y sus características basales al inicio de la ventilación mecánica. Decidimos no tomar en cuenta parámetros de ergotrauma como el poder mecánico, ${ }^{23}$ ya que en últimas revisiones no se ha encontrado relación al pronóstico de los pacientes con SDRA, ${ }^{24}$ y es un cálculo complejo difícil de realizar al pie de la cama del paciente.

Este estudio tiene como fortaleza el seguimiento diario de los pacientes bajo ventilación mecánica, y su evolución de la mecánica ventilatoria. No existe un estudio sobre el comportamiento de la mecánica ventilatoria en estos pacientes. Sin embargo, este estudio tiene varias limitaciones. Primero, el tamaño de la muestra es pequeña, no se logró realizar análisis de mortalidad.

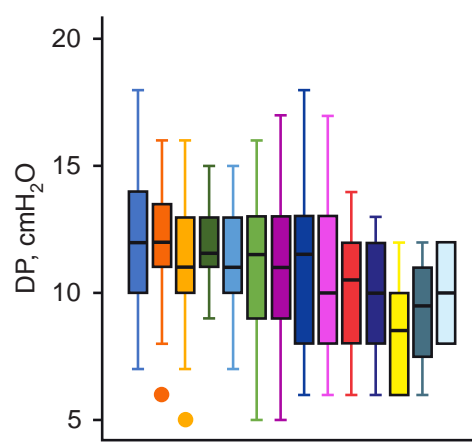

Et

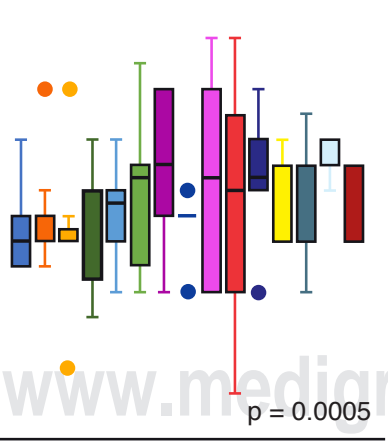

VM prolongada

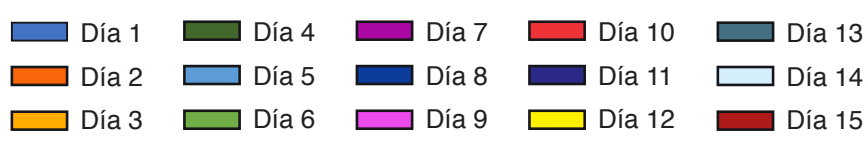

Figura 2: Comportamiento cronológico comparativo de la driving pressure entre los pacientes con extubación temprana versus ventilación prolongada. $\mathrm{DP}=$ driving pressure; $\mathrm{Et}$ = extubación temprana; $\mathrm{VM}$ prolongada = ventilación mecánica prolongada.
Segundo, el seguimiento de los pacientes posterior al 15 de mayo fueron 30 días, no hay información de los pacientes después del seguimiento. Tercero, no tomamos en cuenta información microbiológica de sobreinfección, ya que no todos los casos presentaron cultivos positivos, tomamos en cuenta parámetros inflamatorios que elevan la sospecha de sobreinfección (procalcitonina). Cuarto, no tomamos en cuenta tratamientos utilizados para hipoxemia grave, como prono y uso de bloqueantes neuromusculares, ya que todos recibieron dicho tratamiento por protocolo de tratamiento del centro asistencial.

\section{CONCLUSIONES}

La hipoxemia en pacientes bajo ventilación mecánica por COVID-19 se debe a dos causas: aumento de cortocircuitos pulmonares y desequilibrio de la ventilación/ perfusión pulmonar. Los factores más importantes en ventilación mecánica en su evolución cronológica para evitar eventos no deseados son mantener una DP menor a 13 y un volumen tidal menor a $7.5 \mathrm{~mL} / \mathrm{kg}$. Aunque los hallazgos de los niveles de PEEP no fueron estadísticamente significativos, recomendamos no disminuir su presión por la relación estrecha con la DP en los primeros cinco días.

\section{REFERENCIAS}

1. Hariri L, Hardin CC. COVID-19, angiogenesis, and ARDS endotypes. N Engl J Med. 2020;383(2):182-183.

2. Carsana L, Sonzogni A, Nasr A, Rossi RS, Pellegrinelli A, Zerbi $\mathrm{P}$, et al. Pulmonary post-mortem findings in a series of COVID-19 cases from northern Italy: a two-centre descriptive study. Lancet. 2020;20(10):1135-1140.

3. Ackermann M, Verleden SE, Kuehnel M, Haverich A, Welte T, Laenger $\mathrm{F}$, et al. Pulmonary vascular endothelialitis, thrombosis, and angiogenesis in Covid-19. N Engl J Med. 2020;383(2):120-128.

4. Langford BJ, So M, Raybardhan S, Leung V, Westwood D, MacFadden DR, et al. Bacterial co-infection and secondary infection in patients with COVID-19: a living rapid review and meta-analysis. Clin Microbiol Infect. 2020;26(12):1622-1629.

5. Chen N, Zhou M, Dong X, Qu J, Gong F, Han Y, et al. Epidemiological and clinical characteristics of 99 cases of 2019 novel coronavirus pneumonia in Wuhan, China: a descriptive study. Lancet [Internet]. 2020;395(10223):507-513. Available from: http://dx.doi.org/10.1016/S0140-6736(20)30211-7

6. Li X, Ma X. Acute respiratory failure in COVID-19: is it "typical" ARDS? Crit Care. 2020;24(1):198.

7. Ranieri VM, Rubenfeld GD, Thompson BT, Ferguson ND, Caldwell E, Fan E, et al. Acute respiratory distress syndrome: The Berlin definition. JAMA. 2012;307(23):2526-2533.

8. Gattinoni L, Chiumello D, Rossi S. COVID-19 pneumonia: ARDS or not? Crit Care. 2020;24(1):154.

9. Ferrando C, Suarez-Sipmann F, Mellado-Artigas R, Hernández $M$, Gea A, Arruti E, et al. Clinical features, ventilatory management, and outcome of ARDS caused by COVID-19 are similar to other causes of ARDS. Intensive Care Med. 2020;46(12):2200-2211.

10. von Elm E, Altman DG, Egger M, Pocock SJ, Gotzsche PC, Vandenbroucke JP. Declaración de la Iniciativa STROBE (Strengthening the Reporting of Observational studies in Epidemiology): directrices para la comunicación de estudios observacionales. Gac Sanit. 2008;22(2):144-150. 
11. Bunney PE, Zink AN, Holm AA, Billington CJ, Kotz CM. Orexin activation counteracts decreases in nonexercise activity thermogenesis (NEAT) caused by high-fat diet. Physiol Behav. 2017;176:139-148.

12. Amato MB, Barbas CS, Medeiros DM, Magaldi RB, Schettino GP, Lorenzi-Filho G, et al. Effect of a protective-ventilation strategy on mortality in the acute respiratory distress syndrome. N Engl J Med. 1998;338(6):347-354.

13. Acute Respiratory Distress Syndrome Network, Brower RG, Matthay MA, Morris A, Schoenfeld D, Thompson BT, et al. Ventilation with lower tidal volumes as compared with traditional tidal volumes for acute lung injury and the acute respiratory distress syndrome. N Engl J Med. 2000;342(18):1301-1308.

14. Amato MB, Meade MO, Slutsky AS, Brochard L, Costa EL, Schoenfeld DA, et al. Driving pressure and survival in the acute respiratory distress syndrome. N Engl J Med. 2015;372(8):747755.

15. Grasselli G, Zangrillo A, Zanella A, Antonelli M, Cabrini L, Castelli A, et al. Baseline characteristics and outcomes of 1591 patients infected with SARS-CoV-2 admitted to ICUs of the Lombardy region, Italy. JAMA. 2020;323(16):1574-1581.

16. Zavascki AP, Falci DR. Clinical Characteristics of Covid-19 in China. N Engl J Med. 2020;382(19):1859.

17. Bellani G, Laffey JG, Pham T, Fan E, Brochard L, Esteban A, et al. Epidemiology, patterns of care, and mortality for patients with acute respiratory distress syndrome in Intensive Care Units in 50 countries. JAMA. 2016;315(8):788-800.

18. Mekontso-Dessap A, Castelain V, Anguel N, Bahloul M, Schauvliege F, Richard C, et al. Intensive Care Med. 2002;28(3):272-277.

19. Roy TK, Secomb TW. Theoretical analysis of the determinants of lung oxygen diffusing capacity. J Theor Biol. 2014;351:1-8.
20. Harman D. The aging process: Major risk factor for disease and death. Proc Natl Acad Sci U S A. 1991;88(12):5360-5363.

21. Masi P, Bagate F, d'Humieres T, Al-Assaad L, Abou Chakra L, Derumeaux G, et al. Is hypoxemia explained by intracardiac or intrapulmonary shunt in COVID-19-related acute respiratory distress syndrome? Ann Intensive Care [Internet]. 2020;10(1):4-6. Available from: https://doi.org/10.1186/ s13613-020-00726-z

22. Riley RL, Cournand A. Analysis of factors affecting partial pressures of oxygen and carbon dioxide in gas and blood of lungs; theory. J Appl Physiol. 1951;4(2):77-101.

23. Gattinoni L, Tonetti T, Cressoni M, Cadringher P, Herrmann $\mathrm{P}$, Moerer $\mathrm{O}$, et al. Ventilator-related causes of lung injury: the mechanical power. Intensive Care Med. 2016;42(10):1567-1575.

24. Coppola S, Caccioppola A, Froio S, Formenti P, De Giorgis V, Galanti $V$ et al. Effect of mechanical power on intensive care mortality in ARDS patients. Crit Care. 2020;24(1):246.

\section{Patrocinios: Ninguno. \\ Relación de conflicto de intereses: Ninguno.}

Correspondencia:

Dr. Alfredo Aisa Álvarez, MSc

Centro Médico ABC.

Av. Carlos Fernández Graef Núm. 154,

Santa Fe, Contadero, 05330,

Cuajimalpa de Morelos,

Ciudad de México, México.

Tel: 55 1103-1600

E-mail: alfredoaisaa@gmail.com 